\title{
Analysis Of Field Synergy In Bottom Heated Lid Driven Cubical Cavity
}

\author{
H.P. Rani ${ }^{1}$ *, V.Narayana ${ }^{1}$, Y. Rameshwar ${ }^{2}$ \\ ${ }^{1}$ Department of Mathematics, National Institute of Technology, Warangal-506004, India \\ ${ }^{2}$ Department of Mathematics, University College of Engineering, Osmania University, Hyderabad-500007, India
}

\begin{abstract}
This study presents an innovative visualization tool for the analysis of the mixed convection in a lid-driven air filled cubical cavity heated from below. The total energy of the flow in the cavity is visualized based on the energy stream functions or energy streamlines. Also the heat transfer enhancement in the cavity is presented with an analogy between conduction and convection, namely, the field synergy principle. Flow is assumed to be driven by the vertical temperature gradient and by the top lid of the cavity, which is assumed to slide on its own plane at a uniform speed. The top and bottom walls are assumed to be isothermal and all other walls are thermally insulated. Non dimensional governing equations of this problem are solved by using the finite volume method. Established open source CFD package OpenFOAM is utilized to investigate the flow with respect to the control parameters arising in the system. The nonlinear terms arising in the governing equations are discretized with the NVD schemes. The convection differencing schemes namely, UPWIND, QUICK, SUPERBEE and SFCD discussed and are used to simulate the flow using MPI code. It is observed that the computational cost for all the differencing schemes get reduced tremendously when the MPI code is implemented. Also SFCD scheme gave the Nuseelt number values close to those available in the literature. Extensive numerical flow visualization is conducted for the Reynolds number $(\operatorname{Re}=100,400,1000)$ and the Richardson number $(\mathrm{Ri}=0.001,1,10)$, which categorize the free and forced convective flow, respectively. It is observed that for a fixed value of Re, as Ri increases, the average Nusselt number $(\overline{N u})$, decreases. This shows that the natural convection starts to prevail with an increasing of Ri. But, for a fixed Ri, as Re increases $(\overline{N u})$ increases and the forced convection mode becomes dominant, leading to a chaotic flow. Plots demonstrating the influences of Re and Ri in terms of the contours of the fluid streamlines, isotherms, vortex corelines, and field synergy principle. The synergy angle of buoyant-aiding flow is high while the buoyant-opposing flow is significantly less than that of forced convection flow.
\end{abstract}

\section{INTRODUCTION}

Mixed convective flows in the cubical cavity have been paid attention extensively in the past few decades both experimentally and numerically due to their applications in solar power collector, food processing, crystal growth, electronic device cooling, high performance building insulation, metal casting, glass production, are to name a few. Some of these solutions are known as the benchmark results and are used as the baseline to investigate the quality of emerging new numerical techniques. Quite a number of numerical experiments on heat transfer flows with free and/or forced convection have been paid attention for the past few decades, such type of two-dimensional (2D) cavity flows examined by numerical modelers [5-8] due its simplicity in obtaining the solutions of the governing equations.

Moallemi and Jang [7] studied the buoyancy effect in a $2 \mathrm{D}$ cavity with respect to $\operatorname{Pr}(0.01 \leq \operatorname{Pr} \leq 50), \operatorname{Re}(102 \leq$ $\operatorname{Re} \leq 2000)$ and constant Grashof number (Gr). They observed that contribution of free convection assists the forced convection with the presence of top moving wall. Similar cavity problem analyzed by Prasad et al. [10] numerically for different aspect ratios (AR) in which the top wall is maintained at a higher temperature in comparison with the bottom wall and vice versa. They noticed that when $\mathrm{AR}=0.5$ and 1.0 and for negatively increasing values of $\mathrm{Gr}$, convection is strong inside the cavity. When AR increased to 2 and for $\mathrm{Gr}=-105$, Hopf bifurcation showed its presence. When $A R$ value is further increased to 10, Sharif [11] showed that along the heated sliding wall of the cavity the $\mathrm{Nu}$ shoots up and it decreases rapidly towards the right side of the wall. However, on the other side $\mathrm{Nu}$ shows the oscillatory behavior due to the occurrence of a separation bubble at the cold wall.

Kosef and Street $[5,6]$ described the flow inside a closed cavity exhibits 3D characteristics due to the presence noslip boundary conditions. Similar numerical problem studied by Iwatsu and Hyun [4], in which the top moving wall is maintained at higher temperature than that the bottom wall. They analyzed the temperature distribution

\footnotetext{
* Corresponding author: hprani@nitw.ac.in
} 
in this conduction dominated air filled container by enhancing the convective flow for $102 \leq \mathrm{Re} \leq 2000$ and $0 \leq \mathrm{Ri} \leq 10$. Mohammad and Viskanta [8] numerically studied both $2 \mathrm{D}$ and $3 \mathrm{D}$ cases and they observed the presence of convective cells which are formed due to the bottom heating with lid driven motion. For small values of $\mathrm{Ri}(=0.1)$ i.e., the large forced convection, Benkacem et al. [1] noticed, the average $\mathrm{Nu}$ increases with a cavity inclination. In FVM the convection term is discritized based Central Differencing (CD), which is of second order accurate. However, combination of the explicit time integration and $\mathrm{CD}$ creates an unconditionally unstable solutions. In order to attain stability, differencing schemes of first order accurate have been introduced. The unsatisfactory behavior of first order schemes with respect to the boundedness, the combined spatial and temporal discretization introduces an unnecessary dependence of the solution on the timestep used to create it. Hence it is important to obtain bounded numerical solutions while solving the transport equations. Thus, a good differencing scheme must balance between boundedness and accuracy.

Apart from the issues of accuracy and boundedness, which are essential for accurate calculations, modern differencing schemes are also required to be convergent and computationally inexpensive. The issue of computational cost includes both the additional face-byface operations required to determine the weighting factors in Total Variation Diminishing (TVD) and Normalised Variable Diagram (NVD) schemes and the additional effort required to get solutions for steady-state problems. With the development of NVD, the accuracy and boundendness of differencing schemes has been improved at the expense of convergence. For this reason, still there is a need to analyse the convection discretisation schemes. In this paper numerical examples are given using the high order convection differencing schemes, namely, Upwind Difference, QUICK, SUPERBEE from a family of TVD schemes and SFCD from a family of NVD schemes. They are compared with respect to their accuracy and computational time. The SIMPLE algorithm is used to deal with the pressurevelocity coupling. The Gauss-Seidel method of iterative procedure is used to solve the system of algebraic equations. The gird independent non-uniform mesh is used such that the near wall eddies is captured accurately. The results are validated with those results available in the literature and also shown that the distributed memory parallel processors supports greater potential CPU speedup.

To describe flow field and thermal field characteristics from the enormous output data, apart from the traditional plotting tools such as isotherms and streamlines, the new technique of convection heat transfer is called field synergy principle Guo et al. [2, 3] are employed in the present study.

\section{PHYSICAL SYSTEM AND GOVERNING EQUATIONS}

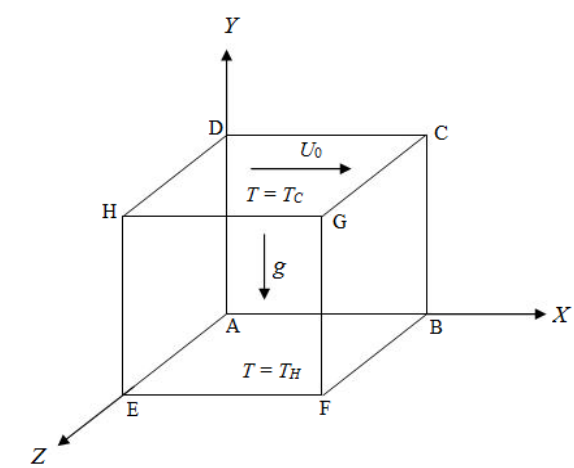

Fig. 1. 3D cubical driven cavity of length $\mathrm{L}$

Considered 3D model is schematically shown in Fig. 1. The bottom wall of the cavity is assumed to be maintained at a high temperature $\left(T_{H}\right)$ than the top moving wall $\left(T_{C}\right)$ with $\Delta T=T_{H}-T_{C}>0$ whereas the other remaining walls are adiabatic. The steady-state non-dimensional governing equations of this schematic are as follows:

$\Delta . \boldsymbol{V}=0$

$(\boldsymbol{V} . \nabla) \boldsymbol{V}=-\nabla p+\frac{1}{R e} \cdot \nabla^{2} \boldsymbol{V}+R i . T^{*} \boldsymbol{e}$

$(\boldsymbol{V} . \nabla) T^{*}=\frac{1}{R e P r} \cdot \nabla^{2} T^{*}$

where notations are explained in the Nomenclature. The boundary conditions are

$\boldsymbol{V}=(1,0,0)$ at $Y=1$ and $\boldsymbol{V}=0$ at $Y=0$,

$\forall X=0,1 ; \forall Z=0,1$.

$T^{*}=1$ at $Y=0 ; T^{*}=0$ at $Y=1$;

$\frac{\partial T^{*}}{\partial X}=0$, at $X=0,1 ; \frac{\partial T^{*}}{\partial Z}=0, Z=0,1$.

The Nusselt number along the hot wall is given by $N u=$ $-\left(\frac{\partial T^{*}}{\partial Y}\right)_{Y=0}$.

\section{NUMERICAL METHOD \& VALIDATION}

The finite volume method is adapted to discretize the Eqs. (1-3). Established open source CFD package Open FOAM, has been utilized. The geometry, volume, boundary and initial conditions are set in the buoyantBoussinesqSimpleFoam. The spatial derivatives are discretized by employing the second order upwind linearization technique. The Laplacian and Divergent terms are discretized by the Gauss linear and SFCD schemes, respectively. The CGS method was employed to accelerate the convergence.

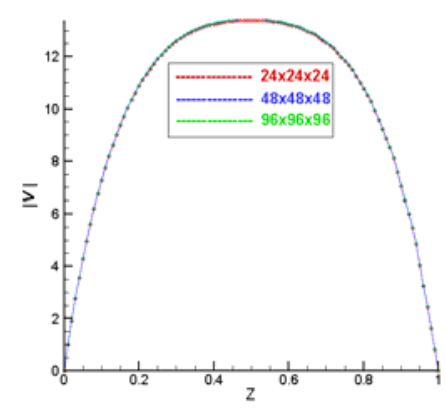

Fig. 2. Velocity magnitude $|\mathrm{V}|$ at $\mathrm{XY}$ mid plane (Grid independent test for $\mathrm{Re}=1000, \mathrm{Ri}=0.001$.) 


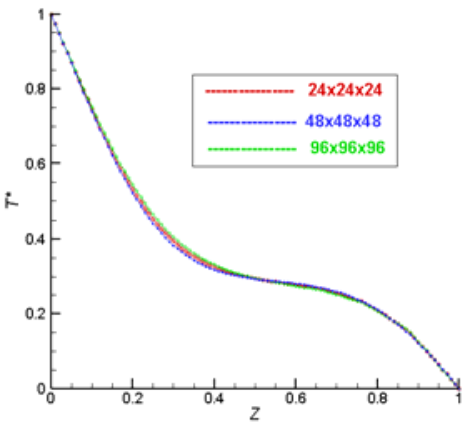

Fig. 3. (b) Temperature $T^{*}$ at $\mathrm{XY}$ mid plane (Grid independent test for $\mathrm{Re}=1000, \mathrm{Ri}=0.001$.)

Non uniform mesh structure is adapted so as to capture the near wall eddies. In order to obtain an economical and reliable grid system for the computations, a mesh refinement study is conducted with three different meshes namely $24^{3}, 48^{3}$ and $96^{3}$. For these meshes the simulated $|V|$ and $T^{*}$ on the $X Y$ - mid plane for $R e=100$ and $R i=$ 0.001, are shown in Fig. 2 (a) and (b), respectively. The simulated results using $48^{3}$ and $96^{3}$ nonuniformly stretched mesh system do not show a meaningful difference, but the result with $24^{3}$ mesh system shows a deviation from those of the above two mesh systems. Thus, the $48^{3}$ mesh resolution was employed in the present study.

Table 1. Validation of $\overline{N u}$ at the hot wall for different Re and $\mathrm{Ri}$

\begin{tabular}{|c|l|c|c|c|}
\hline \multirow{2}{*}{$\begin{array}{l}R i \\
R e\end{array}$} & & 0.001 & 1 & 10 \\
\hline \multirow{3}{*}{100} & Present work & 1.802 & 1.298 & 1.078 \\
\cline { 2 - 5 } & Iwatsu et al. [4] & 1.82 & 1.33 & 1.08 \\
\cline { 2 - 5 } & Ouertatani et al. [9] & 1.836 & 1.348 & 1.092 \\
\hline \multirow{3}{*}{400} & Present work & 3.912 & 1.518 & 1.15 \\
\cline { 2 - 5 } & Iwatsu et al. [4] & 3.99 & 1.50 & 1.17 \\
\cline { 2 - 5 } & Ouertatani et al. $[9]$ & 3.964 & 1.528 & 1.130 \\
\hline
\end{tabular}

As for the validation of the current work, the present simulated results are compared with Iwatsu and Hyun [4], Ouertatani et al. [9] in terms of $(\overline{N u})$ at the hot wall and tabulated in Table 1. There is an excellent agreement between the simulated and existing results. It can be observed that for a fixed value of Re, as Ri increases, $(\overline{\mathrm{Nu}})$ decreases. This shows that the natural convection starts to prevail with an increasing of Ri. But, as Re increases $(\overline{N u})$ increases and the forced convection mode becomes dominant, leading to a chaotic flow.

\subsection{Convection differencing schemes}

The nonlinear terms arising in the Eqs.(1)-(3) are discretized with the NVD schemes. The convection differencing schemes namely, UPWIND, QUICK, SUPERBEE and SFCD are discussed below and are used to simulate the flow using MPI code.

\subsubsection{SUPERBEE scheme}

In this method a higher-order differencing scheme is expressed as a sum of the first-order bounded upwind differencing scheme (UD) and a "limited" higher order correction $(\mathrm{HO})$

$\Phi_{f}=(\Phi)_{U D}+\psi\left[(\Phi)_{H O}-(\Phi)_{U D}\right]$

where $(\Phi)_{H O}$ denotes the face value of $\Phi$ for the selected higher order scheme and $\Psi$ represents the flux limiter.

$r=\frac{\Phi_{C}-\Phi_{U}}{\Phi_{D}-\Phi_{C}}$

Hence $\psi=\psi(\mathrm{r})$. The points U, C and D are selected based on the direction of flow on the face $f$. In this method the limiter function $\Psi(\mathrm{r})=\operatorname{Max}[0, \min (2 \mathrm{r}, 1), \min (\mathrm{r}, 2)]$.

\subsubsection{SFCD scheme}

The SFCD scheme, employs the boundedness of the UDS and the accuracy of CDS. This scheme is a central differencing scheme with a native filter to remove unphysical extrema whenever they would arise. Basically this filter is a local shifting towards the UDS in such zones. Thus, in this method, blending of CDS and UDS will be done by a blending factor $\gamma$, ranging from 0 to 1 depending on computed face, e as defined as

$\Phi_{e}{ }^{S F C D}=\gamma_{e} \Phi_{e}^{C D S}+\left(1-\gamma_{e}\right) \Phi_{e}^{U D S}$

The blending factor will be as close as possible to unity in case of steep gradients in order to get sharp discontinuities; while in other more uniform zones stability is reached with $\gamma$ close to zero.

Table 2. Test comparison of convection differencing schemes when $\mathrm{Re}=100$ and $\mathrm{Ri}=10$.

\begin{tabular}{|c|c|c|c|}
$\begin{array}{c}\text { Convection } \\
\text { Schemes }\end{array}$ & $\overline{N u}$ & $\begin{array}{c}\text { CPU time with } \\
\text { MPI (s) }\end{array}$ & $\begin{array}{c}\text { CPU time } \\
\text { without MPI (s) }\end{array}$ \\
\hline UPWIND & 0.687 & 68245 & 259200 \\
\hline QUICK & 0.7214 & 84568 & 349880 \\
\hline SUPERBEE & 0.9596 & 94698 & 383483 \\
\hline SFCD & 1.078 & 92589 & 374901 \\
\hline
\end{tabular}

Table 2 shows the results in terms of $(\overline{N u})$ and they are compared with respect to their CPU time. It is observed that the computational cost for all the differencing schemes get reduced tremendously when the MPI code is implemented. Also SFCD scheme gave the $\overline{N u}$ results close to those available in the literature.

\section{RESULTS AND DISCUSSION}

In this section, the simulated results are plotted with respect to flow topology using the streamlines, isotherms, and field synergy with respect to Re and Ri.

\subsection{Flow topology: fluid streamlines and vortex corelines}

In this study, an analysis is performed from a large quantity of simulated data to understand the nature of the flow topology for different values of the control parameters using fluid stream-lines, limiting streamlines 
and vortex corelines. The limiting streamlines are the fluid streamlines considered in the very neighborhood of the end walls. The velocity gradient eigen-mode method is used to extract these vortex core lines. The vortex corelines are regarded as the main signature of the vortical flow. Figure 3 shows the fluid streamlines on the mid $X Y$ plane, limiting fluid streamlines near the adiabatic vertical walls, and vortex core lines for different combinations of Ri and Re as mentioned earlier in the previous paragraph.

From Fig. 3, it can be observed that for all combinations of $(R e, R i)$, the fluid flow is characterized by a primary rotating unicellular vortex occupying the bulk of the cavity generated by the movement of the lid and corner vortices near the bounding bottom walls. The structure, which is termed the "corner vortex," is originated from the shear and pressure forces acting on the re-circulating fluid along with the no-slip condition imposed by the presence of physical lateral boundaries.

The vertical corelines, surrounded by the spiraling streamlines as shown in Fig. 3 reveal their origin/end points are located on the adiabatic walls. This shows that critical points where the wall shear stress vanishes are located on the bounding walls (Tony et al. [12]). The spiraling character of the flow seemed to be more pronounced and became stronger with increasing $R e$. It can be noted that, when $R i$ or $R e$ increases, the primary vortex migrates from the top sliding wall to center of the cavity. This movement of the primary vortex is more pronounced for the natural convection dominated flows as seen from the vortex core lines drawn along the primary vortex arising in the system. Also it can be seen that for small values of $R e$ the core line reveals almost a straight line between the vertical adiabatic walls and the center of the cavity. This implies that the recirculation of the fluid cells takes place uniformly throughout the cavity. When $R e$ (or the velocity of the lid) increases, the recirculating flow located at the center of the cavity moves down from its original position in comparison with the flow formed close to the adiabatic wall. This result can be further visualized from the vortex core drawn along the recirculating flow regions of the cavity. Further increment of Re leads the primary vortex at the center of the cavity try to reach the bottom wall of the cavity. Also two small recirculating flows or corner vortices showed their appearance at the bottom corners, adjacent to the adiabatic walls.

When $R i=1$, as Re increases (Fig. 3(b, e, h)), the position of the vortex core line gets more bended, and also one right recirculating flow is formed on the plane at $\mathrm{Z}=0.5$. When $R i=10$, as $R e$ increases (see Fig. 3(c, f, i)), also only a circulating flow is formed at the bottom right corner of the cavity in the mid $X Y$ plane at $\mathrm{Z}=0.5$. It is due to the fact that the velocity of the lid causes the center of the primary vortex to move from the top to bottom wall (Fig. 3(c, f, i)).

\subsection{Isotherms}

The impact of $R e$ and $R i$ on the temperature contours is illustrated in Fig. 4. The isotherms are drawn on the cross sectional symmetry plane $Z=0.5$. It can be seen that, when $G r<10^{4}$, temperature contours are almost vertical in the vicinity of isothermal walls. This result implies still conduction mechanism prevails and convection not yet started. When $G r>10^{4}$ isotherms change its shape from almost vertical to horizontal and the boundary layers showed their presence adjacent to isothermal walls. This trend does not appear near the adiabatic walls. When $R i$ is small (forced convection) and as Re increases from 100 to 400 , vertical isotherms appears due to increase of velocity of the upper lid. From Figs. 4(a,d) displays buoyancy driven convection is dominated by the mechanically driven forced convection.

\subsection{Field synergy angle}

Local intersection angle is given by (Guo et al. (1998)

$\beta=\cos ^{-1}\left(\frac{V \cdot \nabla T}{|V||\nabla T|}\right)$

The average intersection angle $\left(\beta_{m}\right)$ of the cavity is given by

$\beta_{m}=\frac{\sum \beta_{i, j, k} d v_{i, j, k}}{\sum d v_{i, j, k}}$

Where $d v_{i, j, k}$ is the volume element. From Eq. (9) it is observed that synergy determines the strength of the convective heat transfer.

Figure 5 shows the contours field synergy $\beta$ value for various $R i$ and $R e$ at $\mathrm{X}=0.1,0.5$ and 0.9 planes. When $R e$ is small, i.e near to the boundaries, the field synergy increases with the increase of $R i$ (Fig. 5(a-c)) this is due to conduction where heat transfer rate also increases but the angle between temperature gradient and velocity vector is decreases at the walls. Whereas the middle portion of the cavity synergy is less but synergy angle is more. From Fig.5(d-f) it is observed that more synergy at the middle portion of cavity due to convection of heat transfer whereas worse near to the boundaries.

Figure 6 shows the variation of synergy angle under different values of $R i$ and $R e$. As Re increases with increase of $R i$, synergy angle also increases, i.e., the synergy between velocity vector and temperature field becomes worse, which leads to slight increase of average Nusselt number $\overline{N u}$ which signifies the rate of heat transfer across the surface. Since the moving fluid always enhances convective heat transfer.

\section{CONCLUSIONS}

In the present study flow is assumed to be driven by the vertical temperature gradient and by the top lid of the cavity, which is assumed to slide on its own plane at a uniform speed. The top and bottom walls are assumed to be isothermal and all other walls are thermally insulated. Non dimensional governing equations of this problem are solved computationally. The nonlinear terms arising in the governing equations are discretized with the NVD schemes, namely, UPWIND, QUICK, SUPERBEE and SFCD. It is observed that the computational cost for all the differencing schemes get reduced tremendously 
when the MPI code is implemented. Also SFCD scheme gave the Nusselt number values close to those available in the literature. The investigation of top wall moving bottom heated cubical cavity explores symmetric nature near the boundaries. When $R i$ is small with increase of $R e$, two emerging eddies formed at the bottom corners of the cavity due to the upper sliding lid movement, whereas one right eddy formed at high $R i$ because of velocity of the lid, which causes the principal vortex moved from top to bottom and also because of the forced convection $\overline{N u}$ increases. As Re increases, synergy angle increases for distinct $R i$ values. So the synergy between temperature and velocity gets worse. The synergy angle of buoyant-aiding flow is high while the buoyant-opposing flow is significantly less than that of forced convection flow when $R i=1$. This gives the relation between temperature field and velocity in buoyant-aiding flow, which is at the deteriorating situation and leading to increasing average Nusselt number.

\section{NOMENCLATURE}

$\begin{array}{ll}C_{p} & \text { Specific heat } \\ e & \text { Unit vector } \\ \bar{E} & \text { energy flux density vector } \\ g & \text { gravitational acceleration } \\ G r & \text { Grashof number }\left(=\frac{g \beta(\Delta T) L^{3}}{v^{3}}\right) \\ K & \text { Thermal conductivity } \\ L & \text { Length of the cavity } \\ N u & \text { Local Nusselt number } \\ \overline{N u} & \text { Average Nusselt number } \\ p & \text { non dimensional pressure } \\ P r & \text { Prandtl number }\left(=\frac{v}{\alpha}\right) \\ R e & \text { Reynolds number }\left(=U_{0} h / v\right) \\ R i & \text { Richardson number }\left(=G r . R e^{-2}\right) \\ T & \text { Dimensional temperature } \\ T^{*} & \text { Dimensionaless temperature }\left(=\frac{T-T_{C}}{\Delta T}\right) \\ U_{0} & \text { Top wall velocity } \\ V & \text { Dimensionless velocity vector } \\ X, Y, Z & \text { Cartesian coordinates } \\ \beta & \text { field synergy angle between } V \text { and the } \\ & \text { temperature gradient } \nabla T \\ \beta_{m} & \text { Average included angle of } \beta_{\mathrm{f}} \\ \Delta T & \text { characteristic temperature difference } \\ \nabla T^{*} & \text { dimensionless temperature gradient } \\ v & \text { kinematic viscosity } \\ \sigma & \text { viscous stress tensor } \\ \rho & \text { density }\end{array}$

\section{KEYWORDS}

Magnetohydrodynamics, Field synergy, Heat transfer, Rotation, Taylor number, Chandrasekhar number and Advanced numerical method.

\section{References}

1. N. Benkacem, N, B, Cheikh, B.B. Beya, Threedimensional analysis of mixed convection in a differentially heated lid-driven cubic enclosure, $J$. Appl. MechEng., 4, 3 (2015).

2. G. Guo, D. Li, B. Wang, A novel concept for convective heat transfer enhancement. Int. J. Heat Mass Transfer. 41, 2221-2225 (1998).

3. Z. Guo, W. Tao, R. Shah, The field synergy (coordination) principle and its applications in enhancing single phase convective heat transfer. Int. J. Heat Mass Transfer. 48 1797- 1807 (2005).

4. R. Iwatsu, J. M. Hyun, Three dimensional drivencavity flows with a vertical temperature gradient. Int. J. Heat Mass Transfe, 38 3319-3328 (1995)..

5. J. R. Koseff, R. L. Street, On end wall effects in a lid driven cavity flow. J. Fluids Eng., 106, 385-389 (1984).

6. J. R. Koseff, R. L. Street, Visualization studies of a shear driven three dimensional recirculating Flow. $J$. Fluids Eng., 106, 21-29 (1984).

7. M. K Moallemi, K. S. Jang, Prandtl number effects on laminar mixed convection heat transfer in a liddriven cavity. Int. J. Heat Mass Transfer. 35, 18811892(1992).

8. A. A. Mohammad, R.Viskanta, Laminar flow and heat transfer in Rayleigh-Benard convection with shear, Phys. Fluids. 4, 2131-2140(1992)..

9. N.Ouertatani, N.B. Cheikh, B. B. Beya, T. Lili, C. Antonio, Mixed convection in a double lid-driven cubic cavity, Int. J. Thermal Sciences, Vol. 48, 1265 1272(2009).

10. Y. S. Prasad, M. K. Das, Hopf bifurcation in mixed flow inside a rectangular cavity. Int. J. Heat Mass Transfer. 50, 3583-3598 (2007).

11. M. A. R. Sharif, Laminar mixed convection in shallow inclined driven cavities with hot moving lid on top and cooled from bottom. App.Ther. Eng, 27,1036-1042 (2007)..

12. Tony, W.H. Sheu., H. P. Rani, Teu-Chia Tan., S. F. Tsai, Multiple states, topology and bifurcations of natural convection in a cubical cavity, Comput. \& Fluids. 37, 1011-1028(2008).

\section{Greek letters}

$\alpha \quad$ coefficient of thermal diffusion

$\beta \quad$ coefficient of thermal expansion

\section{Abbrevations}

MPI Message Passing Interface 

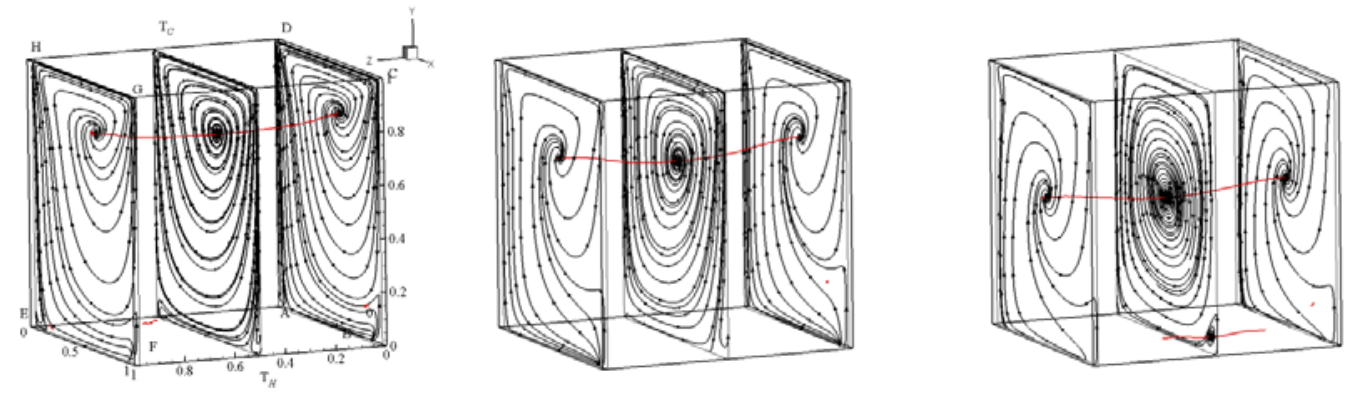

(a) $R i=0.001, R e=100$

(b) $R i=1, R e=100$

(c) $R i=10, R e=100$
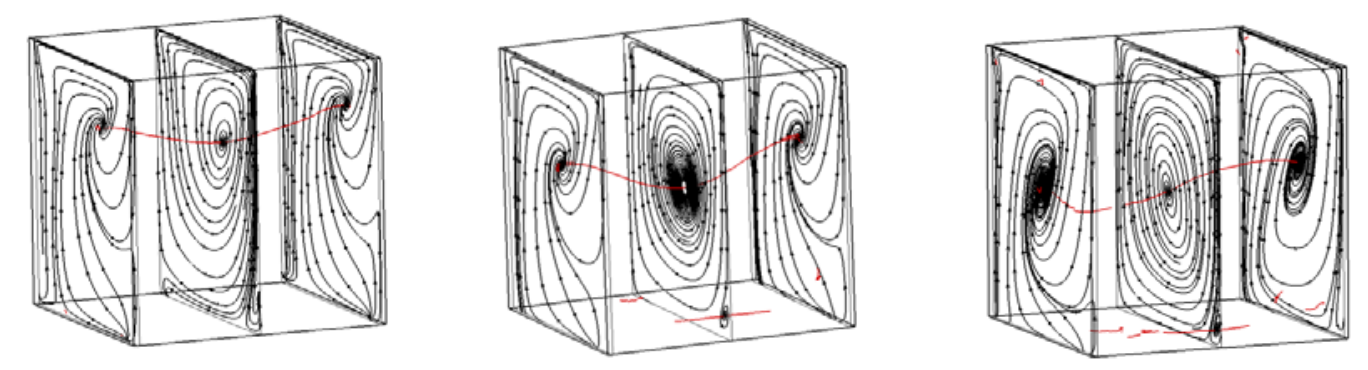

(d) $R i=0.001, R e=400$

(e) $R i=1, R e=400$

(f) $R i=10, R e=400$

Fig. 3 The simulated vortex corelines, limiting streamline(boundaries) and streamlines(center)

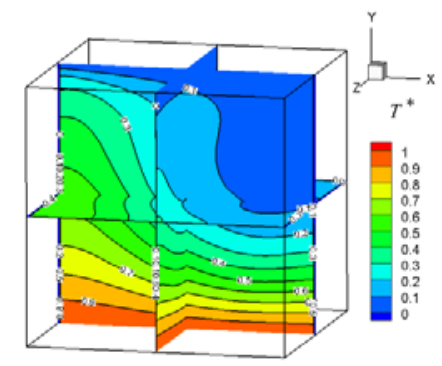

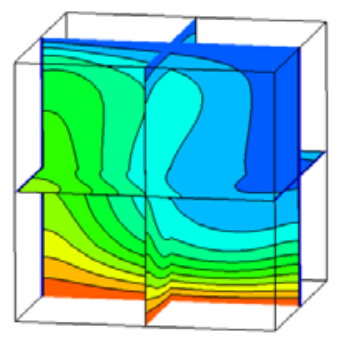

(b) $R i=1, R e=100$

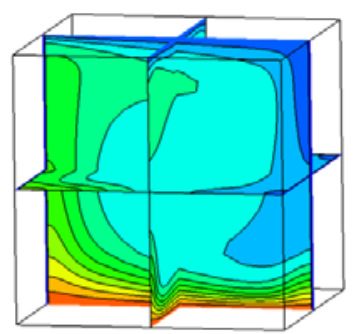

(e) $R i=1, R e=400$

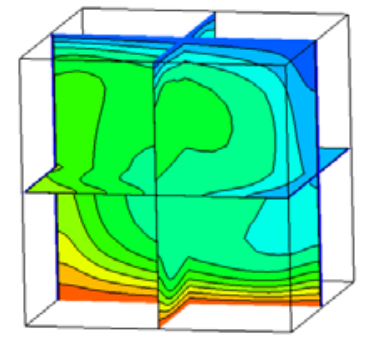

(c) $R i=10, R e=100$

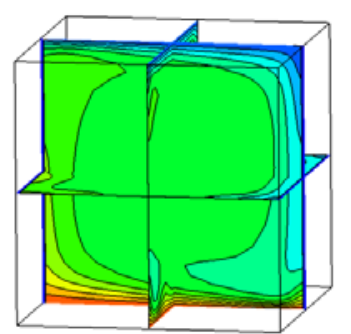

(f) $R i=10, R e=400$

(d) $R i=0.001, R e=400$

Fig. 4 Simulated isotherms for different $R i$ and $R e$ 


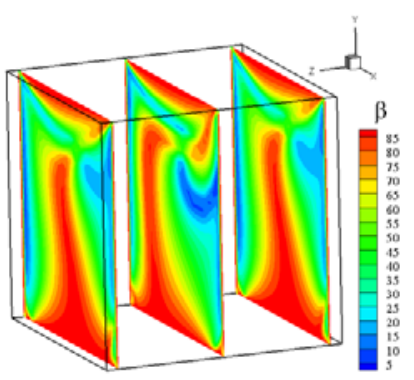

(a) $R i=0.001, R e=100$

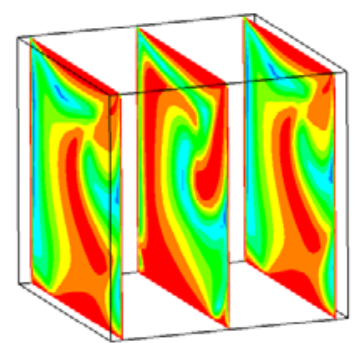

(d) $R i=0.001, R e=400$

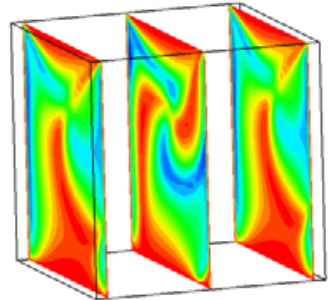

(b) $R i=1, R e=100$

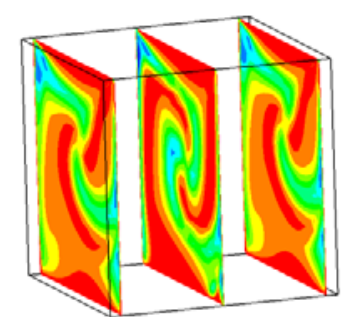

(e) $R i=1, R e=400$

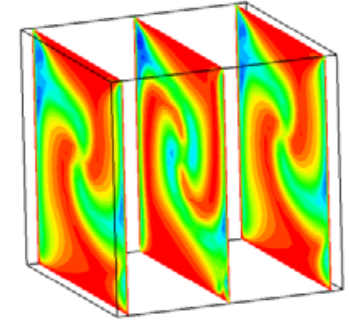

(c) $R i=10, R e=100$

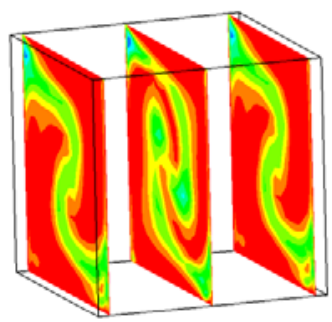

(f) $R i=10, R e=400$

Fig. 5 Field synergy at $\mathrm{X}=0.1,0.5$ and 0.9 for different $R i$ and $R e$

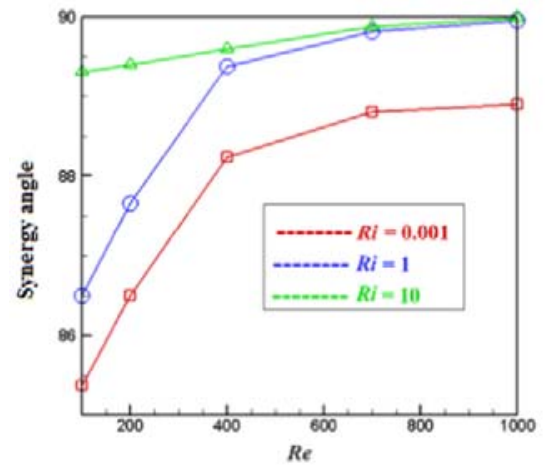

Fig. 6 The simulated field synergy $\left(\beta_{m}\right)$ for different $R e$ and $R i$, the color contours denotes the field synergy and black lines denotes the streamlines. 